\title{
Immune Checkpoint and Ovarian Cancer
}

\section{Bose $\mathrm{CK}^{*}$}

Consultant Gynaecological Oncologist, NSC Bose Cancer Research Institute, Kolkata

${ }^{*}$ Corresponding author: Bose CK, Consultant Gynaecological Oncologist, NSC Bose Cancer Research Institute Kolkata, 8D, MSG Ln, Kolkata 700006, Tel: 9830114880, E-mail: ckbose@hotmail.com

Citation: Bose CK (2017) Immune Checkpoint and Ovarian Cancer. J Gynecol Res 3(1): 104. doi: 10.15744/2454-3284.3.101

Received Date: September 17, 2016 Accepted Date: May 23, 2017 Published Date: May 24, 2017

\begin{abstract}
Many scientists and biotechnology companies had given up on the idea of cancer immunotherapy in 1990s. Almost a decade after first detection of T cell suppression effect of CTLA4 the identity of its antibody was established. While they are found effective in many cancers including melanoma, lung cancer etc. immune checkpoint inhibitors have lent an important measure to manage recurrent and refractory ovarian cancer. This subject needs constant updating specially for students of ovarian cancer who are looking at this avenue of cure with much hope. A search for immune checkpoint in ovarian cancer till date was done through pubmed (40 publications, important are cited here) and Google including FDA website to enumerate recent trial effort in this field for recurrent ovarian cancer. Whereas Pembrolizumab is having highest number of trials all pathways are tried, newer combinations like one with PARP inhibitors are emerging. As a new development this subject is experiencing rapid progress and multiple avenues are opening up. However, there are many hurdles to overcome.
\end{abstract}

Keywords: CTLA4; Immunotherapy in ovarian cancer

\section{Introduction}

Although the idea, called "cancer immunotherapy", is very appealing and has previously been shown to work in several mouse models of cancer, it has in general been very difficult to translate cancer immunotherapy approaches in human. Because of this frustration, by the 1990s, many scientists and biotechnology companies had given up on the idea of cancer immunotherapy. Eight years after first detection T cell suppression effect of CTLA4 the identity of the antibody molecule was established. Antibody to CTLA4 could increase T cell which in turn destroys cancer cell. This started a completely new age of tumor immunology. Besides CTLA4 many immune checkpoints are now shown which pave new ways in manipulation of immunological control over malignant tumor cells. It has lent an important measure to manage especially recurrent and refractory cancers and those cancers where there is an unmet need like recurrent melanoma, renal cell carcinoma and recurrent ovarian cancer. As a new development this subject is experiencing rapid progress and multiple avenues are opening up. Though there are many hurdle to overcome this needs constant updating specially for students of ovarian cancer who are looking at it with much hope.

\section{Early proof of $\mathrm{T}$ cell involvement in ovarian cancer}

More than three decades back T cell infiltration was noticed in ovarian cancers [1]. In 2003 Zhang et al appreciated their role in improved survival. They observed at least $60 \%$ benefit in 5 year survival in a cohort of 74 patients. They were treated to obtain complete clinical response after debulking and platinum-based therapy. CD ${ }^{+} \mathrm{T}$ cells within their tumor and IFN- $\gamma$ plus macrophagederived chemokines made the difference [2]. Reason of such heterogeneity is still unclear though subject has grown hugely and gained much strength. Regulatory T-cell subsets (CD4+) had confusing immune suppressing role. However, improved survival is noticed in patients who had higher numbers of intraepithelial CD8+ T cells compared with patients without intraepithelial CD8+ T cells (median survival 55 vs 26 months) [3,4]. Strong positive correlation was observed between levels of CD8+ T cells and granzyme B within tumors [5]. MHC related interferon regulatory factor (IRF)-1 and metastasis related chemokine receptor (CXCR) are the two genes differentially expressed in tumors with high versus low CD8+ T-cell infiltration. Heterogeneity in the tumor microenvironment among patients with EOC, and various immune cell populations those have been associated positively or negatively with clinical prognosis, included tumor-infiltrating lymphocytes (TILs), [6] myeloid-derived suppressor cells [7], and tumor-associated macrophages [8].

\section{Immune checkpoints in ovarian cancer}

Tumour infiltrating lymphocytes (TILs) express the negative regulatory immune receptor, programmed cell death 1 (PD-1), [9] which were upregulated on T-cell activation and suppresses T-effector functions. Several cellular populations, including cancer 
cells and tumor-associated myeloid cells, express its ligand PD-L1 [10-13]. Expression of PD-L1 by tumors has been associated with decreased intraepithelial TILs and poor overall survival in EOC [13].

In an immune-competent murine model of EOC, PD-1 and PD-L1 blockade has led to eradication of tumors through the expected reprogramming of the tumor microenvironment, [14] which suggested potential benefit from PD-1/PD-L1 inhibition for patients with EOC. The attenuation of T cell function by cytotoxic T lymphocyte-associated antigen 4 (CTLA-4) was also noticed in EOC [15]. Although the binding of peripheral membrane proteins B7-1 or B7-2 to CD28 provides an important costimulatory signal, the engagement of CTLA- 4 by these ligands induced cell cycle arrest and diminished cytokine production [16-18].

\section{Checkpoint Inhibitors in ovarian cancer}

Documented effort to test ovarian cancer started with Hodi et al [19] who gave two patients a single infusion and then nine cases [20] upto 11 infusion of $3 \mathrm{mg} / \mathrm{kg}$ ipilimumab after an autologous ovarian tumor cell vaccine transduced with granulocytemacrophage colony-stimulating factor (GVAX). Only three patients had SD of $>2$ months.

A phase 2 clinical trial of ipilimumab in relapsed platinum-sensitive ovarian cancer with measurable disease is ongoing though not recruiting any more (NCT01611558). Some trials either in post transplantation or along with other checkpoint molecules like nivolumab and pembrolizumab are recruiting. There are other two trials which are either suspended or terminated. The PD-1 antagonist nivolumab is tested at 1 or $3 \mathrm{mg} / \mathrm{kg}$ every 2 weeks in 18 patients with relapsed platinum-resistant disease regardless of PD-L1 expression; there was a $17 \%$ overall response rate (ORR) and a $44 \%$ disease control rate $(\mathrm{DCR}=\mathrm{CR}+\mathrm{PR}+\mathrm{SD})$, with 2 $\mathrm{CR}, 1 \mathrm{PR}$, and 5 patients with SD [21] A phase $1 \mathrm{~b}$ study tested the PD-1 antagonist pembrolizumab at $10 \mathrm{mg} / \mathrm{kg}$ every $2 \mathrm{meeks}$ in 26 patients with heavily treated PD-L1+ ovarian cancer chemotherapy [22]. There was a durable ORR of $11.5 \%$, and a DCR of $34.6 \%$, with 1 CR, 2 PR, and 6 patients with SD. Now pembrolizumab has highest number of clinical trials in ovarian cancer. The PD-L1 antagonist avelumab was given at $10 \mathrm{mg} / \mathrm{kg}$ every 2 weeks in a phase $1 \mathrm{~b}$ study of 75 patients with platinum-resistant or chemotherapy-refractory ovarian cancer regardless of PD-L1 expression [23] with an ORR of 10.7\%, and a DCR of 54.7\%. A phase 1 study of another PD-L1 antagonist, BMS-936559, revealed one objective response in 17 ovarian cancer patients [24]. A summary of trials in ovarian cancer is given in Table 1.

As a whole benefit in OS with ICIs was significant in both younger and older patients with a cut-off age of 65-70 years in a total of 5265 patients from nine RCTs of ICI [25]. When patients are dichotomized into younger and older groups with an age cut-off of 65-70 years, ICIs improved OS in both younger (HR, 0.75; 95\% CI, 0.68-0.82) and older (HR, 0.73; 95\% CI, 0.62-0.87) groups. An improvement in PFS was observed in younger (HR, 0.58; 95\% CI, 0.40-0.84) and older (HR, 0.77; 95\% CI, 0.58-1.01) patients. Subgroup analyses according to ICI and tumor type showed a consistent survival benefit in both younger and older groups except for the subgroup of older patients treated in 4 trials of anti-programmed cell death protein-1 (PD-1) monoclonal antibody (HR, 0.86; 95\% CI, 0.41-1.83).

These drugs generally contain warnings with regard to increased risks of severe immune-mediated inflammation of the lungs, the colon, the liver, the kidneys (with accompanying kidney dysfunction), as well as immune-mediated hypothyroidism and hyperthyroidism.

\begin{tabular}{|c|c|c|}
\hline 1. & Ipilimumab & $\begin{array}{l}\text { 1. Ipilimumab Monotherapy in Recurrent Platinum-sensitive Ovarian Cancer. Active, not recruiting. Has Results Phase II Study } \\
\text { 2. Nivolumab With or Without Ipilimumab in Treating Patients With Persistent or Recurrent Epithelial Ovarian, Primary Peri- } \\
\text { toneal, or Fallopian Tube Cancer Suspended } \\
\text { 3. Safety Study of MGA271 in Combination With Ipilimumab in Refractory Cancer Recruiting } \\
\text { 4. A Study of Nivolumab by Itself or Nivolumab Combined With Ipilimumab in Patients With Advanced or Metastatic Solid } \\
\text { Tumors Recruiting } \\
\text { 5. Monoclonal Antibody Therapy in Treating Patients With Ovarian Epithelial Cancer, Melanoma, Acute Myeloid Leukemia, } \\
\text { Myelodysplastic Syndrome, or Non-Small Cell Lung Cancer Terminated } \\
\text { 6. Ipilimumab After Allogeneic Stem Cell Transplant in Treating Patients With Persistent or Progressive Cancer Completed } \\
\text { 7. Safety Study of MGA271 in Combination With Pembrolizumab in Refractory Cancer Recruiting }\end{array}$ \\
\hline 2. & Tremelimumab & $\begin{array}{l}\text { 1. PARP-inhibition and CTLA-4 Blockade in BRCA-deficient Ovarian Cancer Recruiting } \\
\text { 2. Durvalumab and Tremelimumab in Combination With First-Line Chemotherapy in Advanced Solid Tumors Recruiting } \\
\text { 3. A Phase } 1 \text { Study to Evaluate MEDI } 4736 \text { in Combination With Tremelimumab Recruiting } \\
\text { 4. Study of Tremelimumab Alone or Combined With Olaparib for Patients With Persistent EOC (Epithelial Ovarian, Fallopian } \\
\text { Tube or Primary Peritoneal Carcinoma) Recruiting }\end{array}$ \\
\hline 3. & Nivolumab & $\begin{array}{l}\text { 1. A Study of WT1 Vaccine and Nivolumab For Recurrent Ovarian Cancer Recruiting } \\
\text { 2. A Dose Escalation and Cohort Expansion Study of Anti-CD27 (Varlilumab) and Anti-PD-1 (Nivolumab) in Advanced } \\
\text { Refractory Solid Tumors. Recruiting } \\
\text { 3. A Study of the Safety, Tolerability, and Efficacy of Epacadostat Administered in Combination With Nivolumab in Select } \\
\text { Advanced Cancers. Recruiting } \\
\text { 4. Nivolumab With or Without Ipilimumab in Treating Patients With Persistent or Recurrent Epithelial Ovarian, Primary } \\
\text { Peritoneal, or Fallopian Tube Cancer. Suspended } \\
\text { 5. Study of FPA008 in Combination With Nivolumab in Patients With Selected Advanced Cancers Recruiting } \\
\text { 6. A Phase } 1 \text { Study of AM0010 in Patients With Advanced Solid Tumors Recruiting } \\
\text { 7. A Study of Nivolumab by Itself or Nivolumab Combined With Ipilimumab in Patients With Advanced or Metastatic Solid } \\
\text { Tumors Recruiting }\end{array}$ \\
\hline
\end{tabular}




\begin{tabular}{|c|c|c|}
\hline 4. & Pembrolizumab & $\begin{array}{l}\text { 1. Pembrolizumab in Combination With Chemotherapy in Frontline Ovarian Cancer Not yet recruiting } \\
\text { 2. Pembrolizumab/Carboplatin/Taxol in Epithelial Ovary Cancer Not yet recruiting Phase II: } \\
\text { 3. Study of Niraparib in Combination With Pembrolizumab (MK-3475) in Patients With Triple-negative Breast Cancer or } \\
\text { Ovarian Cancer (KEYNOTE-162)Recruiting } \\
\text { 4. A Study of Pembrolizumab With Standard Treatment in Patients With Recurrent Platinum-resistant Ovarian Cancer Recruiting } \\
\text { 5. Efficacy and Safety Study of Pembrolizumab (MK-3475) in Women With Advanced Recurrent Ovarian Cancer (MK-3475-100/ } \\
\text { KEYNOTE-100) Recruiting } \\
\text { 6. Dose Dense Paclitaxel With Pembrolizumab (MK-3475) in Platinum Resistant Ovarian Cancer Recruiting } \\
\text { 7. ACP-196 Alone and in Combination With Pembrolizumab in Subjects With Recurrent Ovarian Cancer (KEYNOTE191) Recruiting } \\
\text { 8. A Combination Clinical Study of PLX3397 and Pembrolizumab To Treat Advanced Melanoma and Other Solid Tumors Recruiting } \\
\text { 9. Pembrolizumab and Ziv-aflibercept in Treating Patients With Advanced Solid Tumors Recruiting } \\
\text { 10. Study of the Effects of Pembrolizumab in Patients With Advanced Solid Tumors Recruiting } \\
\text { 11. A Study of Pembrolizumab on the Tumoral Immunoprofile of Gynecologic Cancers Not yet recruiting } \\
\text { 12. Study of CM-24 (MK-6018) Alone and In Combination With Pembrolizumab (MK-3475) in Participants With Selected } \\
\text { Advanced or Recurrent Malignancies Recruiting } \\
\text { 13. Safety Study of MGA271 in Combination With Pembrolizumab in Refractory Cancer Recruiting } \\
\text { 14. Pembrolizumab in Subjects With Incurable Platinum-Refractory Germ Cell Tumors Recruiting } \\
\text { 15. A Phase } 1 / 2 \text { Study Exploring the Safety, Tolerability, and Efficacy of Pembrolizumab (MK-3475) in Combination With } \\
\text { Epacadostat (INCB024360) in Subjects With Selected Cancers (INCB 24360-202 / MK-3475-037 / KEYNOTE-037/ ECHO-202) } \\
\text { Recruiting } \\
\text { 16. A Phase } 1 \text { Study of AM0010 in Patients With Advanced Solid Tumors Recruiting } \\
\text { 17. Study of Pembrolizumab Plus Chemotherapy in Patients With Advanced Cancer (PembroPlus) Recruiting } \\
\text { 18. MK-3475 Immunotherapy in Endometrial Carcinoma Recruiting } \\
\text { 19. Phase IB of Selinexor in Combination With Standard Chemotherapy Recruiting } \\
\text { 20. Phase } 1 \text { Study of Intradermal LV305 in Patients With Locally Advanced, Relapsed or Metastatic Cancer Expressing NY-ESO-1 } \\
\text { Recruiting }\end{array}$ \\
\hline 5. & Atezolizumab & $\begin{array}{l}\text { 1. Anti-programmed Cell Death-1 Ligand } 1 \text { (aPDL-1) Antibody Atezolizumab, Bevacizumab and Acetylsalicylic Acid in } \\
\text { Recurrent Platinum Resistant Ovarian Cancer Not yet recruiting } \\
\text { 2. A Study of Atezolizumab Administered in Combination With Bevacizumab and/or With Chemotherapy in Participants With } \\
\text { Locally Advanced or Metastatic Solid Tumors Recruiting }\end{array}$ \\
\hline 6. & Durbalumab & $\begin{array}{l}\text { 1. Matched Paired Pharmacodynamics and Feasibility Study of Durvalumab in Combination With Chemotherapy in Frontline } \\
\text { Ovarian Cancer Not yet recruiting } \\
\text { 2. A Phase } 1 / 2 \text { Study of Motolimod (VTX-2337) and MEDI } 4736 \text { in Subjects With Recurrent, Platinum-Resistant Ovarian Cancer } \\
\text { for Whom Pegylated Liposomal Doxorubicin (PLD) is Indicated Recruiting } \\
\text { 3. Phase } 1 \text { and } 2 \text { Study of MEDI4736 in Combination With Olaparib or Cediranib for Advanced Solid Tumors and Recurrent } \\
\text { Ovarian Cancer Recruiting } \\
\text { 4. TPIV200/huFR-1 (A Multi-Epitope Anti-Folate Receptor Vaccine) Plus Anti-PD-L1 MEDI4736 (Durvalumab) in Patients With } \\
\text { Platinum Resistant Ovarian Cancer Recruiting } \\
\text { 5. Durvalumab and Tremelimumab in Combination With First-Line Chemotherapy in Advanced Solid Tumors Recruiting } \\
\text { 6. MEDI4736 (Durvalumab) in Patients With Brain Metastasis From Epithelial-derived Tumors Not yet recruiting } \\
\text { 7. Study of Azacitidine and Durvalumab in Advanced Solid Tumors Not yet recruiting } \\
\text { 8. A Phase } 1 \text { Study to Evaluate MEDI4736 in Combination With Tremelimumab Recruiting } \\
\text { 9. A Phase I/II Study of MEDI4736 in Combination With Olaparib in Patients With Advanced Solid Tumors .Recruiting }\end{array}$ \\
\hline 7. & Avelumab & $\begin{array}{l}\text { 1. A Study Of Avelumab Alone Or In Combination With Pegylated Liposomal Doxorubicin Versus Pegylated Liposomal } \\
\text { Doxorubicin Alone In Patients With Platinum Resistant/Refractory Ovarian Cancer (JAVELIN Ovarian 200) Recruiting } \\
\text { 2. Avelumab in Previously Untreated Patients With Epithelial Ovarian Cancer (JAVELIN OVARIAN 100) Recruiting } \\
\text { 3. Avelumab in Metastatic or Locally Advanced Solid Tumors (JAVELIN Solid Tumor) Recruiting }\end{array}$ \\
\hline 8. & BMS-936559 & $\begin{array}{l}\text { 1. A Dose Escalation and Cohort Expansion Study of Anti-CD27 (Varlilumab) and Anti-PD-1 (Nivolumab) in Advanced } \\
\text { Refractory Solid Tumors } \\
\text { Intervention: Drug: Combination of varlilumab and nivolumab Recruiting } \\
\text { 2. A Study of CDX-1127 (Varlilumab) in Patients With Select Solid Tumor Types or Hematologic Cancers Active, not recruiting }\end{array}$ \\
\hline 9. & Enoblituzumab & $\begin{array}{l}\text { 1. Safety Study of MGA271 in Combination With Pembrolizumab in Refractory Cancer Recruiting } \\
\text { 2. Safety Study of MGA271 in Combination With Ipilimumab in Refractory Cancer Recruiting } \\
\text { 3. Study of Immune Response Modifier in the Treatment of Breast, Ovarian, Endometrial and Cervical Cancers Completed Has } \\
\text { Results } \\
\text { 4. BMS_PD-L1_onco : Assessment of the PD-L1 Protein as a Biomarker in Oncology and Hematology Recruiting }\end{array}$ \\
\hline 10. & Epacadostat & $\begin{array}{l}\text { 1. Study of DPX-Survivac Vaccine Therapy and Epacadostat in Patients With Recurrent Ovarian Cancer Recruiting } \\
\text { 2. Safety and Efficacy of CRS-207 With Epacadostat in Platinum Resistant Ovarian, Fallopian, or Peritoneal Cancer Recruiting } \\
\text { 3. Epacadostat Before Surgery in Treating Patients With Newly Diagnosed Stage III-IV Epithelial Ovarian, Fallopian Tube, or } \\
\text { Primary Peritoneal Cancer Active, not recruiting } \\
\text { 4.A Study of the Safety, Tolerability, and Efficacy of Epacadostat Administered in Combination With Nivolumab in Select } \\
\text { Advanced Cancers m,Intervention: Drug: Nivolumab + Epacadostat Recruiting } \\
\text { 5. A Phase 1/2 Study Exploring the Safety, Tolerability, and Efficacy of Pembrolizumab (MK-3475) in Combination With } \\
\text { Epacadostat (INCB024360) in Subjects With Selected Cancers (INCB 24360-202 / MK-3475-037 / KEYNOTE-037/ ECHO-202) } \\
\text { Recruiting } \\
\text { 6. DEC-205/NY-ESO-1 Fusion Protein CDX-1401, Poly ICLC, and IDO1 Inhibitor INCB024360 in Treating Patients With } \\
\text { Ovarian, Fallopian Tube, or Primary Peritoneal Cancer in Remission Recruiting }\end{array}$ \\
\hline 11. & Pidilizumab & 1. CT-011 and p53 Genetic Vaccine for Advanced Solid Tumors Withdrawn \\
\hline
\end{tabular}

Table 1: Immune checkpoints in ovarian cancer trials 
In clinical trials for melanoma, the following side effects occurred in more than $10 \%$ of subjects and more frequently than with chemotherapy alone: rash and itchy skin, cough, upper respiratory tract infections, and peripheral edema. Other clinically important side effects with less than $10 \%$ frequency were ventricular arrhythmia, inflammation of parts of the eye (iridocyclitis), infusion-related reactions, dizziness, peripheral and sensory neuropathy, peeling skin, erythema multiforme, vitiligo, and psoriasis. In clinical trials for lung cancer, the following side effects occurred in more than $10 \%$ of subjects and more frequently than with chemotherapy alone: fatigue, weakness, edema, fever, chest pain, generalized pain, shortness of breath, cough, muscle and joint pain, decreased appetite, abdominal pain, nausea and vomiting, constipation, weight loss, rash, and itchy skin. Levels of electrolytes and blood cells counts were also disrupted.

\section{Conclusion}

It is evident from discussion that much obstacles are remaining in establishing immuno therapy in ovarian cancer as response rate is low and no FDA approval is in the offing. Expectedly Professor Maurie Markman has commented at the 33rd Annual Chemotherapy Foundation Symposium "The checkpoint inhibitors are not ready for prime time yet in ovarian cancer. It's not because there's evidence that they don't work - it's just that there's no evidence at all," But that will in no way undermine this important effort of treating first the relapse and refractory cases. It will certainly and find out the hindrance and to make best use of developed knowledge to find ways to subvert shortcoming of this important measure of treatment.

\section{Reference}

1. Haskill S, Becker S, Fowler W, Walton L (1982) Mononuclear-cell infiltration in ovarian cancer. I. Inflammatory-cell infiltrates from tumour and ascites material. Br J Cancer 45: 728-36.

2. Zhang L, Conejo-Garcia JR, Katsaros D, Gimotty PA, Massobrio M, et al. (2003) Intratumoral T cells, recurrence, and survival in epithelial ovarian cancer. N Engl J Med 348: 203-13.

3. Sato E, Olson SH, Ahn J, Bundy B, Nishikawa H, et al. (2005) Intraepithelial CD8+ tumor-infiltrating lymphocytes and a high CD8+/regulatory T cell ratio are associated with favorable prognosis in ovarian cancer. Proc Natl Acad Sci U S A 102: 18538-43.

4. Leffers N, Vermeij R, Hoogeboom BN, Schulze UR, Wolf R, et al. (2012) Long-term clinical and immunological effects of p53-SLP ${ }^{\circledR}$ vaccine in patients with ovarian cancer. Int J Cancer 130: 105-12.

5. Milne K1, Köbel M, Kalloger SE, Barnes RO, Gao D, et al. (2009) Systematic analysis of immune infiltrates in high-grade serous ovarian cancer reveals CD20, FoxP3 and TIA-1 as positive prognostic factors. PLoS One 4: E6412.

6. Curiel TJ, Coukos G, Zou L, Alvarez X, Cheng P, et al (2004) Specific recruitment of regulatory T cells in ovarian carcinoma fosters immune privilege and predicts reduced survival. Nat Med 10: 942-9.

7. Cho H, Hur HW, Kim SW, Kim SH, Kim JH, et al (2009) Pre-treatment neutrophil to lymphocyteratio is elevated in epithelial ovarian cancer and predicts survival after treatment. Cancer Immunol Immunother 58: 15-23.

8. Zhang M, He Y, Sun X, Li Q, Wang W, et al (2013) A high M1/M2 ratio of tumor-associatedmacrophages is associated with extended survival in ovarian cancer patients. J Ovarian Res 7: 19.

9. Matsuzaki J, Gnjatic S, Mhawech-Fauceglia P, Beck A, Miller A, et al (2010) Tumor-infiltrating NY-ESO-1-specific CD8+ T cells are negatively regulated by LAG3 and PD-1 in human ovarian cancer. Proc Natl Acad Sci U S A 107: 7875-80.

10. Abiko K, Mandai M, Hamanishi J, Yoshioka Y, Matsumura N, et al (2013) PD-L1 on tumor cells is induced inascites and promotes peritoneal dissemination of ovarian cancer through CTL dysfunction. Clin Cancer Res 19: 1363-74.

11. Liu Y, Zeng B, Zhang Z, Zhang Y, Yang R (2008) B7-H1 on myeloid-derived suppressor cells in immune suppression by a mouse model of ovarian cancer. Clin Immunol 129: 471-81.

12. Curiel TJ, Wei S, Dong H, Alvarez X, Cheng P, et al (2003) Blockade of B7-H1 improves myeloid dendritic cell-mediated antitumor immunity. Nat Med 9: 562-7.

13. Hamanishi J, Mandai M, Iwasaki M, Okazaki T, Tanaka Y, et al (104) Programmed cell death 1 ligand 1 and tumor-infiltrating CD8 T lymphocytes are prognostic factors of human ovarian cancer. Proc Natl Acad Sci U S A 104: 3360-5.

14. Duraiswamy J, Freeman GJ, Coukos G (2013) Therapeutic PD-1 pathway blockade augments with other modalities of immunotherapy T-cell function to prevent immune decline in ovarian cancer. Cancer Res 73: 6900-12.

15. Preston CC1, Goode EL, Hartmann LC, Kalli KR, Knutson KL (2011) Immunity and immune suppression in human ovarian cancer. Immunotherapy 3: 539-56. 16. Thompson CB, Allison JP (1997) The emerging role of CTLA-4 as an immune attenuator. Immunity 7: 445-50.

17. Doyle AM, Mullen AC, Villarino AV, Hutchins AS, High FA, et al. (2001) Induction of cytotoxic T lymphocyte antigen 4 (CTLA-4) restricts clonal expansion of helper T cells. J Exp Med 194: 893-902.

18. Salomon B, Bluestone JA (2001) Complexities of CD28/B7: CTLA-4 costimulatory pathways in autoimmunity and transplantation. Annu Rev Immunol 19: 225-52.

19. Hodi FS, Mihm MC, Soiffer RJ, Haluska FG, Butler M, et al. (2003) Biologic activity of cytotoxic T lymphocyte-associated antigen 4 antibody blockade in previously vaccinated metastatic melanoma and ovarian carcinoma patients. Proc Natl Acad Sci USA 100: 4712-7.

20. Hodi FS, Butler M, Oble DA, Seiden MV, Haluska FG, et al. (2008) Immunologic and clinical effects of antibody blockade of cytotoxic T lymphocyte-associated antigen 4 in previously vaccinated cancer patients. Proc Natl Acad Sci USA 105: 3005-10.

21. Hamanishi J, Mandai M, Ikeda T, Minami M, Kawaguchi A, et al. (2014) Efficacy and safety of anti-PD-1 antibody (Nivolumab: BMS-936558, ONO-4538) in patients with platinum-resistant ovarian cancer. J Clin Oncol 32: 5511.

22. Varga A, Piha-Paul SA, Ott PA, Mehnert JM, Berton-Rigaud D, et al. (2015) Antitumor activity and safety of pembrolizumab in patients (pts) with PD-L1 positive advanced ovarian cancer: Interim results from a phase Ib study. J Clini Oncol (Meeting Abstracts) 33): 5510.

23. Disis ML, Patel MR, Pant S, Infante JR, Lockhart AC, et al. (2015) Avelumab (MSB0010718C), an anti-PD-L1 antibody, in patients with previously treated, recurrent or refractory ovarian cancer: A phase Ib, open-label expansion trial. J Clin Oncol 33: 2015. 
24. Brahmer JR, Tykodi SS, Chow LQ, Hwu WJ, Topalian SL, et al. (2012) Safety and activity of anti-PD-L1 antibody in patients with advanced cancer. N Engl J Med 366: 2455-65

25. Nishijima TF, Muss HB, Shachar SS, Moschos SJ (2016) Comparison of efficacy of immune checkpoint inhibitors (ICIs) between younger and older patients: A systematic review and meta-analysis. Cancer Treat Rev 45: 30-7. 